\title{
An unusual case of neuroblastoma: a 17-year-old adolescent presented with bilateral diffuse lung metastasis at initial diagnosis
}

\author{
Çağlar Ödek ${ }^{1}$, Tanıl Kendirli¹, Esra Pekpak², Ayhan Yaman, Başak Adaklı-Aksoy², \\ Handan Dinçaslan², Nurdan Taçyıldız², Emel Ünal ${ }^{2}$, Erdal İnce ${ }^{1}$, Gülsan Yavuz ${ }^{2}$ \\ Divisions of ${ }^{1}$ Pediatric Critical Care and ${ }^{2}$ Pediatric Oncology, Department of Pediatrics, Ankara University Faculty of \\ Medicine, Ankara, Turkey.E-mail: odek@ankara.edu.tr \\ Received: 13 February 2015, Accepted: 8 April 2015
}

\begin{abstract}
SUMMARY: Ödek Ç, Kendirli T, Pekpak E, Yaman A, Adakl1-Aksoy B, Dinçaslan H, Taçyıldız N, Ünal E, İnce E, Yavuz G. An unusual case of neuroblastoma: A 17-year-old adolescent presented with bilateral diffuse lung metastasis at initial diagnosis. Turk J Pediatr 2016; 58: 86-89.

Neuroblastoma (NB) is the most frequently diagnosed neoplasm during infancy and its incidence declines within the first 3-5 years of life. It can be rarely diagnosed in adolescents and young adults. Adolescents have advanced stage of disease, higher frequency of uncommon metastatic sites such as lungs, and worse outcomes. Herein, we describe an unusual case of NB in a 17-yearold adolescent presented with lung metastasis at diagnosis. The patient was diagnosed with stage IV NB. Thorax high-resolution computed tomography (HRCT) scan revealed irregular septal thickening with ground glass opacity consistent with pulmonary parenchymal metastases. After the first cycle of chemotherapy he developed pulmonary hemorrhage and respiratory distress. He required ventilation support and mechanical ventilation was started. Metastatic nodules were determined on second thorax HRCT. We lost the patient due to septic shock and multiple organ failure 2 months after diagnosis. In conclusion, adolescents with NB have unfavorable prognosis. These patients may have lung metastases at diagnosis. Therefore, detailed chest imaging at initial diagnosis is crucial.
\end{abstract}

Key words: neuroblastoma, adolescent, lung metastasis, pulmonary hemorrhage.

Neuroblastoma (NB) is the most common extracranial solid tumor in pediatric population and derived from primordial neural crest cells. It accounts for $8-10 \%$ of all childhood cancers and $15 \%$ of childhood cancer mortality. The annual incidence of NB is about 10 per million live births during infancy ${ }^{1}$. Peak age of incidence is 2 years and approximately $90 \%$ of cases are diagnosed before age $5^{2}$. Only about $3 \%$ of NB is diagnosed in adolescents and young adults ${ }^{3}$.

Neuroblastoma may develop anywhere along the sympathetic neural chain. Sixty-five percent of them occur within the abdomen, with at least half of these tumors arising in the adrenal medulla 4 . About $50 \%$ of the patients will present with metastatic disease. Long bones and skull, bone marrow, liver, lymph nodes, and skin are the most common sites of metastasis. Lung metastases are rare and have been reported to be an unfavorable metastatic site $^{2}$. Herein, we describe an unusual case of NB in a 17-year-old adolescent presented with bilateral diffuse lung metastasis at diagnosis and developed pulmonary hemorrhage.

\section{Case Report}

A previously healthy 17-year-old boy was presented with a chief complaint of right hip and right orbital pain. He had been suffering from hip pain for about a month and orbital pain became prominent for the last 10 days. Physical examination revealed unilateral exophthalmos, periorbital ecchymosis, and tenderness on the right hip joint. Complete blood count revealed: hemoglobin $(\mathrm{Hb}), 9.9$ g/dl; white blood cell (WBC) count, 2900/ $\mathrm{mm}^{3}$ (absolute neuthrophil count, 1900/ $\mathrm{mm}^{3}$ ); platelet count, $81,000 / \mathrm{mm}^{3}$. Lactate 

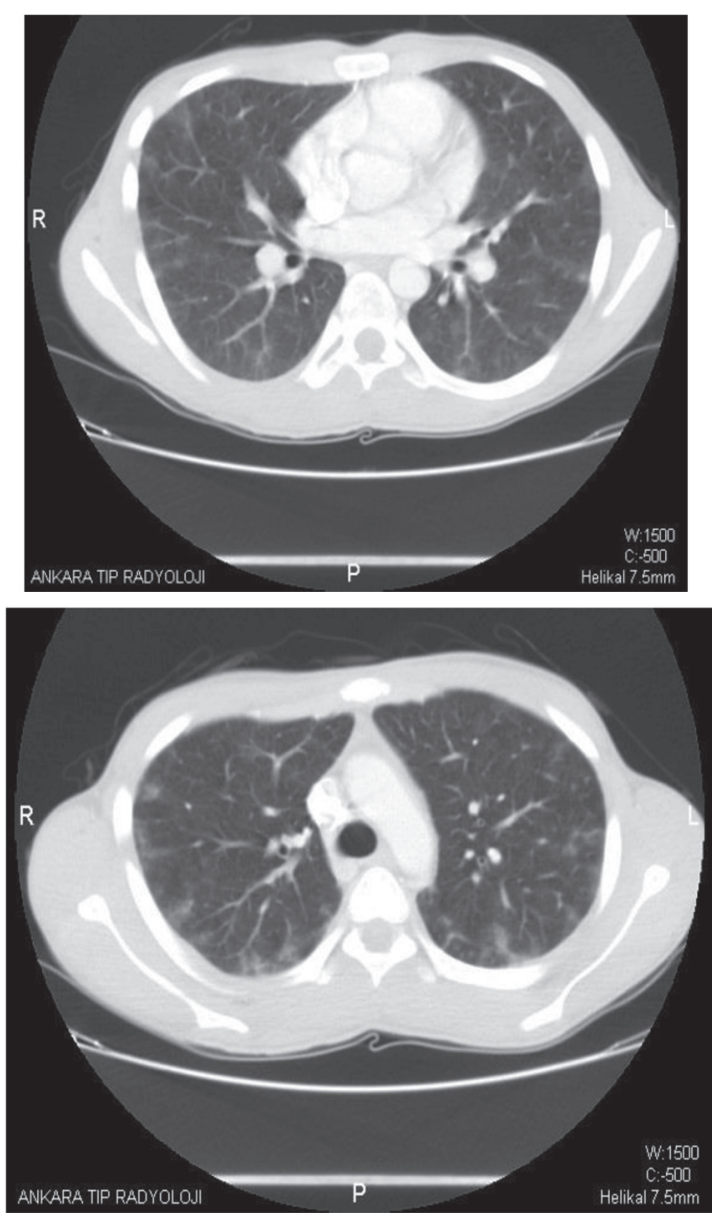

Fig. 1. Thorax HRCT scan findings at diagnosis; septal thickening with ground glass opacity at upper zones and mosaic attenuation at lower zones

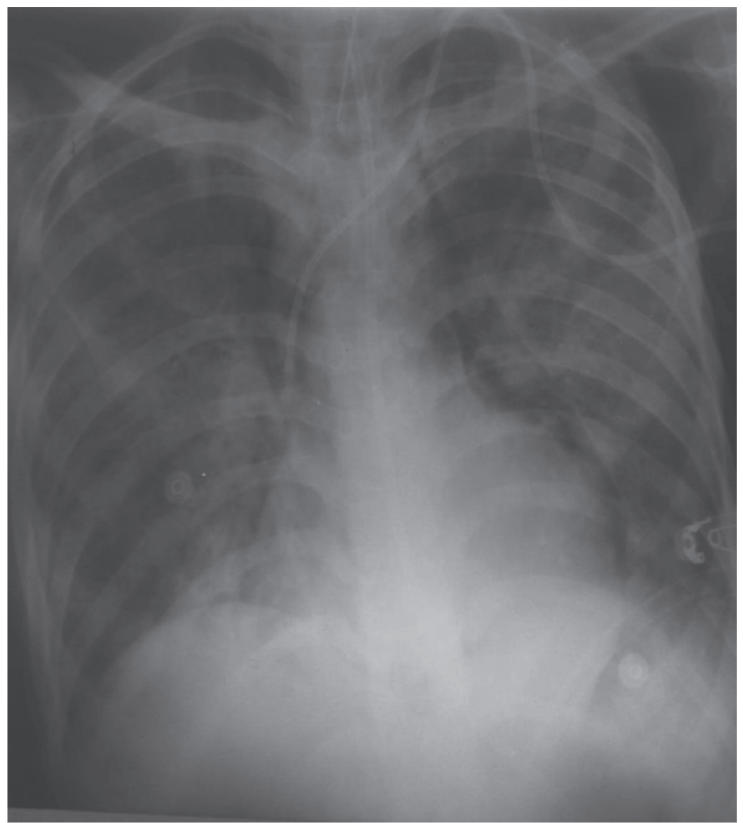

Fig. 2. Chest X-ray at PICU admission; bilateral interstitial infiltrations dehydrogenase (LDH) level was elevated (743 IU/L; normal range, 125-220). He was evaluated radiologically and found to have bony thickening of the superior aspects of the right orbit and multiple calvarial metastases on head computed tomography (CT) scan and positron emission tomography (PET) scan; involvement of sacrum on pelvic CT scan and PET scan; an $8.5 \times 6.5 \mathrm{~cm}$ left adrenal mass and metastatic para-aortic lymph nodes on abdomen CT scan and PET scan; septal thickening with ground glass opacity at upper zones and mosaic attenuation at lower zones on thorax high-resolution CT (HRCT) scan (Fig. 1). Neuron specific enolase and spot urine vanillylmandelic acid levels were elevated (624 $\mu \mathrm{g} / \mathrm{L}$ and $49.9 \mathrm{mg} / \mathrm{L}$, respectively), strongly pointing towards a NB. Bone marrow biopsy revealed infiltration by a malignant small round cell tumor with formation of rosette pattern. Immunstain for chromogranin, a synaptophysin, CD56 were strongly positive. Based on these findings, he was diagnosed with stage IV NB. MYCN amplification, chromosome 1 p deletion, $\mathrm{t}(11 ; 22)$, and $A L K$ gene mutations were negative. Chemotherapy was started, consisting of: vincristin, dacarbazine, ifosfamide and adriamycin (A11 protocol). Fifteen days after initiation of the first cycle of chemotherapy, he developed fatigue, hemoptysis and respiratory distress. He was diagnosed with pulmonary hemorrhage and admitted to our pediatric intensive care unit (PICU). At the time of PICU admission, the level of consciousness was depressed. His vital signs were as follows: heart rate, 129 beats/min; respiratory rate, 27 breaths/min; blood pressure: 114/76 $\mathrm{mmHg}$; and pulse oximetry was $82 \%$ without oxygen supplementation and $93 \%$ with non-rebreathing oxygen mask. Physical examination revealed cyanosis, intercostal and subcostal retractions, and nasal flaring. There were bilateral crackles on chest auscultation. Complete blood count revealed: $\mathrm{Hb}, 6.8 \mathrm{~g} / \mathrm{dl}$; WBC count, $4000 / \mathrm{mm}^{3}$; platelet count, $50,000 / \mathrm{mm}^{3}$. C-reactive protein was $12.8 \mathrm{~g} / \mathrm{dl}$. Coagulation parameters and electrolytes were normal. Chest X-ray revealed bilateral interstitial infiltrations (Fig. 2) and blood gas analysis showed hypoxemia $(\mathrm{pH}$ : 7.46, $\mathrm{PCO}_{2}: 32.7 \mathrm{mmHg}, \mathrm{PO}_{2}: 61.4 \mathrm{mmHg}$, $\mathrm{HCO}_{3}: 24 \mathrm{mEq} / \mathrm{L}, \mathrm{BE}: 0.1, \mathrm{SaO}_{2}: 86 \%$ ). His $\mathrm{PO}_{2} / \mathrm{FiO}_{2}$ ratio was 68. Echocardiography revealed normal cardiac functions. Pediatric Risk of Mortality III (PRISM III) ${ }^{5}$ and Pediatric 

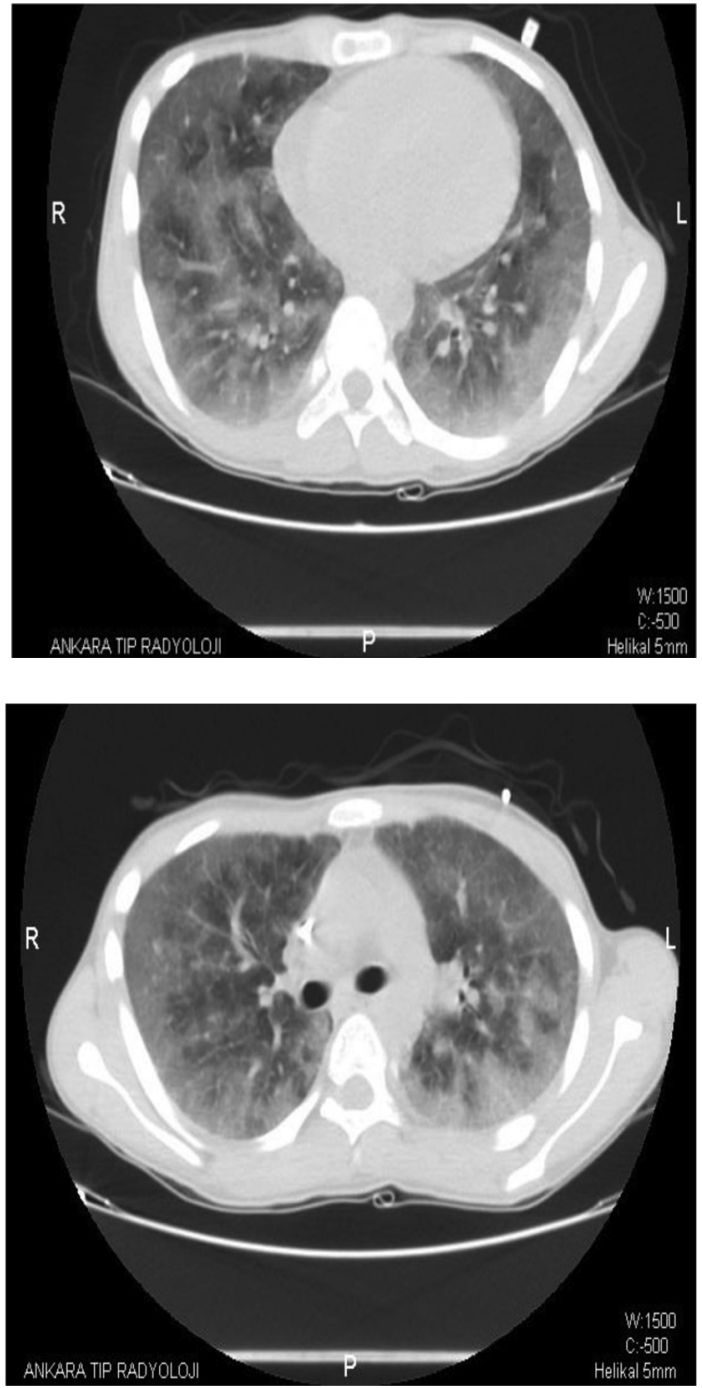

Fig. 3. Thorax HRCT scan findings at PICU admission; crazy paving, diffuse septal thickening with ground glass opacity, and new onset nodules randomly distributed throughout the lungs with a predilection for the upper zones

Logistic Organ Dysfunction (PELOD) ${ }^{6}$ scores were 9 and 11, respectively. We defined acute respiratory distress syndrome (ARDS) using the criteria defined by the American-European Consensus Conference on ARDS ${ }^{7}$. The patient was intubated and mechanical ventilation was started. Addition of positive end-expiratory pressure (PEEP) up to $13 \mathrm{cmH}_{2} \mathrm{O}$ improved his oxygenation. A red blood cell transfusion was given. Meropenem, teicoplanin, clarythromycin, trimethoprim/sulfamethoxazole (TMP-SMX) and caspofungin were started for antibacterial and antifungal coverage. After stabilization of his clinical status, thorax HRCT scan was performed. It revealed crazy paving, diffuse septal thickening with ground glass opacity and new onset nodules pointing towards pulmonary hemorrhage and metastatic disease. Nodules were maximum $5 \mathrm{~mm}$ in diameter and randomly distributed throughout the lungs with a predilection for the upper zones (Fig. 3 ). Tracheal aspirate culture, respiratory virus polymerase chain reaction (PCR) panel, and PCR tests for Pneumocystis jiroveci, cytomegalovirus DNA, and Ebstein-Barr virus DNA obtained at PICU admission were all negative. A few days later the patient developed septic shock and multiple organ failure. He was treated according to the international guidelines for septic shock ${ }^{8}$. However, his clinical status worsened and patient died on PICU day 17. A postmortem lung biopsy was performed and it revealed diffuse pulmonary hemorrhage; pleural, subpleural, and parenchymal metastases of NB.

\section{Discussion}

Neuroblastoma is the most frequently diagnosed neoplasm during infancy and its incidence declines within the first 3-5 years of life $^{2}$. It becomes very rare among adolescents and adults $(<1$ diagnosis per 1 million per year $)^{3}$. Previous studies on NB occuring in older children, adolescents and adults are not homogeneous because of the age groups considered (6 to 52 years), thus results are different ${ }^{9}$. In an Italian study, Conte et al. ${ }^{3}$ reported that adolescents aged 10 to 18 years represented only $2.8 \%$ of the entire NB population (53 over a total of 1866 patients in a 23 year period).

In addition to tumor extention, patient age at diagnosis has been recognized as a powerful indicator of clinical behavior and recent data suggests that a cut-off around 18 months of age might be preferable for the optimal prognostic distinction ${ }^{2}$. However, due to the rarity of NB in adolescent age group, information about the impact of age on survival and characteristics of the disease is scarce. In general, the disease has some peculiarities and a more unfavorable prognosis in adolescent patients ${ }^{9}$. Studies showed that adolescents usually have advanced stage of disease and worse outcomes compared with children with the same disease stage ${ }^{3}$. The frequencies of known biological risk factors such as MYCN amplification and chromosome $1 \mathrm{p}$ deletion are lower ${ }^{9}$. Adolescents with 
NB have a higher frequency of uncommon metastatic sites such as lungs and brain ${ }^{3}$. Similar to previous reports, our patient had an advanced stage of disease and lung metastasis at diagnosis. MYCN amplification and chromosome $1 \mathrm{p}$ deletion were negative.

The lung is the most common site of metastasis in other pediatric extra-cranial solid cancers including Ewing sarcoma, osteosarcoma, and Wilms tumor ${ }^{10}$. Lung metastasis in NB is rare and previous studies reported the incidence of lung metastasis at diagnosis between $0.2 \%$ and $3.7 \%^{11,12}$. Patients with lung metastasis at initial diagnosis appear to have aggressive tumors. DuBois et $\mathrm{al}^{10}$. showed that these patients were more likely to have adrenal primary tumors, elevated LDH levels, MYCN amplified tumors and decreased event-free survival when compared with all children with stage IV NB without lung metastasis. Our patient had an adrenal primary tumor and elevated LDH levels but MYCN amplification was negative.

Various imaging modalities are used in diagnosis and evaluation of disease progression and extention. Detailed chest imaging is important to establish lung metastasis. Imaging studies include chest radiographs, HRCT scan, metaiodobenzylguanidine (MIBG) scan and positron emission tomography scan. DuBois et $\mathrm{al}^{10}$. suggested that the true incidence of lung metastasis in patients with NB might be higher because detailed chest imaging studies are not available for all patients. HRCT is the goldstandard modality for chest imaging because small lesions could be missed on radiographs ${ }^{11}$. Lung metastasis of NB at initial diagnosis included multiple nodules between $5-10 \mathrm{~mm}$ in diameter, parenchymal metastasis, mediastinal lymphadenopathy, pleural thickening and effusion, and pleural-based nodules ${ }^{11}$. In our patient, chest X-ray at PICU admission revealed bilateral diffuse interstitial infiltrations. The differential diagnosis included bacterial and viral infections, pulmonary edema, pulmonary hemorrhage, and metastatic NB. Although the initial thorax HRCT scan showed non-specific septal thickening at upper zones and mosaic attenuation at lower zones, metastatic nodules were determined on the second HRCT scan which was performed at PICU day 2 (45 days after the first one). We suggest that differential diagnosis should include lung metastasis even in patients with non-specific HRCT findings.
In conclusion, $\mathrm{NB}$ occurs rarely during adolescence. Adolescents have advanced stage of disease, a more unfavorable prognosis and a higher frequency of uncommon metastatic sites such as lungs. Therefore, detailed chest imaging at diagnosis is crucial in adolescent patients and thorax HRCT scan is the gold standard modality. Patients with lung metastasis usually have aggressive tumors and these patients may develop pulmonary hemorrhage during or after chemotherapy.

\section{REFERENCES}

1. Brodeur GM, Hogarty MD, Mosse YP, Maris JM. Neuroblastoma. In: Pizzo PA, Poplack DG (eds). Principles and Practice of Pediatric Oncology (6th ed) Philadelphia: Lippincott Williams \& Wilkins; 2011: 886-922.

2. Esiashvili N, Anderson C, Katzenstein HM. Neuroblastoma. Curr Probl Cancer 2009; 33: 333-360.

3. Conte M, Parodi S, De Bernardi B, et al. Neuroblastoma in adolescents: the Italian experience. Cancer 2006; 106: $1409-1417$

4. Maris JM, Hogarty MD, Bagatell R, et al. Neuroblastoma. Lancet 2007; 369: 2106-2120.

5. Pollack MM, Patel KM, Ruttimann UE. PRISM III: an updated Pediatric Risk of Mortality Score. Crit Care Med 1996; 24: 743-752.

6. Leteurtre S, Martinot A, Duhamel A, et al. Validation of the paediatric logistic organ dysfunction (PELOD) score: prospective, observational, multicentre study. Lancet 2003; 362: 192-197.

7. Bernard GR, Artigas A, Brigham KL, et al. The American-European Consensus Conference on ARDS: Definitions, mechanisms, relevant outcomes, and clinical trial coordination. Am J Respir Crit Care Med 1994; 143: 818-824.

8. Dellinger RP, Levy MM, Rhodes A, et al. Surviving sepsis campaign: international guidelines for management of severe sepsis and septic shock: 2012. Crit Care Med 2013; 41: 580-637.

9. Conte M, De Bernardi B, Milanaccio C, et al. Malignant neuroblastic tumors in adolescents. Cancer Lett 2005; 228: 271-274.

10. DuBois SG, London WB, Zhang Y, et al. Lung metastases in neuroblastoma at initial diagnosis: A report from the International Neuroblastoma Risk Group (INRG) Project. Pediatr Blood Cancer 2008; 51: 589-592.

11. Kammen BF, Matthay KK, Pacharn P, et al. Pulmonary metastases at diagnosis of neuroblastoma in pediatric patients: CT findings and prognosis. AJR Am J Roentgenol 2001; 176: 755-759.

12. Cowie F, Corbett R, Pinkerton CR. Lung involvement in neuroblastoma: incidence and characteristics. Med Pediatr Oncol 1997; 28: 429-432. 